\title{
Uncertainty in estimating distances from memory
}

\author{
GABRIEL A. RADVANSKY and LAURA A. CARLSON-RADVANSKY \\ University of Notre Dame, Notre Dame, Indiana \\ and \\ DAVID E. IRWIN \\ University of Illinois at Urbana-Champaign, Urbana, Illinois
}

\begin{abstract}
Magnitude estimations involving spatial characteristics, such as distance, typically show a compressive function when estimates are made from memory. In particular, as the magnitude of a property grows larger and larger, estimates become more and more inaccurate, with increasing underestimates of the actual magnitude. Previous theories have attempted to explain this difference by supposing that magnitude estimation was accomplished through a reperceptual process, in which the errors of perception are magnified, or a transformation process, in which the memory trace undergoes a consistent alteration toward a more schematic form. The present experiments present evidence in support of an uncertainty hypothesis. When subjects are uncertain of the actual value of a distance, they are forced to guess on the basis of the mean distance they encountered, because they are unable to retrieve the information accurately. When they can retrieve the information, they are more certain and their estimates are more accurate. This hypothesis was also extended to integrative conditions in which the subjects were presented with the stimulus display in a piecemeal fashion. In these cases, distance estimates were derived by combining spatial representations. This method of presentation caused distance estimates to become less accurate.
\end{abstract}

Estimations of spatial magnitude, such as distance or area, can be described by a power function (S. S. Stevens \& Galanter, 1957). This claim holds whether the stimulus is perceptually available or must be retrieved from memory (Björkman, Lundberg, \& Tärnblom, 1960; Moyer, Bradley, Sorensen, Whiting, \& Mansfield, 1978; Moyer, Sklarew, \& Whiting, 1978; Thorndyke, 1981) and is consistent with the notion that memory for spatial information bears a second-order isomorphic relationship to that information's structure in the real world (Shepard \& Chipman, 1970). While estimates derived from both perception and memory produce similar psychophysical functions, there are some important differences, such as the exponent of the power function. The purpose of this study was to further investigate the differences between magnitude estimation based on perception and memory.

Psychophysical judgment involving perception can be characterized according to Stevens's law by a power function relating physical magnitude $(\Phi)$ and psychological magnitude $(\Psi)$ and can be stated formulaically as $\Psi=$ $k \Phi^{n}$, where $n$ describes the slope of the function in $\log / \log$

This research was supported in part by NSF Grant SRB-93-09564 awarded to D.E.I., and by NIMH National Research Service Award MH14257 to the University of Illinois while the first author was a postdoctoral trainee in the Quantitative Methods Program of the Department of Psychology, University of Illinois at Urbana-Champaign. The authors would like to thank Nancy Franklin, Jim Howard, and two anonymous reviewers for their comments on earlier versions of this paper. We would also like to thank Steve Weede for his assistance in collecting the data. Please address all correspondence concerning this paper to G. A. Radvansky, Department of Psychology, University of Notre Dame, Notre Dame, IN 46556 (e-mail: gabriel.a.radvansky.1@nd.edu). coordinates for perception estimates and $k$ is a constant scaling factor. For memory psychophysics, the power function is the relation between the physical magnitude and the remembered magnitude $(M)$. The formula then takes the form $M=k^{\prime} \Phi^{n^{\prime}}$, where $n^{\prime}$ is the slope of the function in $\log / \log$ coordinates for memory estimates and $k^{\prime}$ is a constant scaling factor. For the purposes of this paper, $n$ and $n^{\prime}$ will be referred to simply as a function's exponent. The $y$-intercept of the function corresponds to $\log (k)$ and $\log \left(k^{\prime}\right)$, and will be simply referred to as the $y$-intercept.

Typically, the exponent for perception estimates of distance is near unity (e.g., Ekman \& Junge, 1961; S. S. Stevens \& Galanter, 1957; Wiest \& Bell, 1985), although it can be affected by certain factors, such as the number of intervening items (Thorndyke, 1981) or the structural organization of a spatial array (McNamara, Ratcliff, \& McKoon, 1984). In contrast, the exponent for memory estimates is noticeably smaller or compressive (e.g., Algom, Wolf, \& Bergman, 1985; Chew \& Richardson, 1980; Kemp, 1988; Moyer, Bradley et al., 1978; Moyer, Sklarew, \& Whiting, 1978 ; Wiest \& Bell, 1985). In a meta-analysis of 70 studies of distance estimation, Wiest and Bell (1985) found that the average perceptual exponent was 1.08 , whereas the average memory exponent was 0.91 . This phenomenon is reminiscent of the famous poster depicting a New Yorker's view of the world. In that picture, the distance to places within New York are represented more or less accurately, but as places become further and further removed, they are depicted as being closer together than they actually are, as well as smaller (and less significant).

Several hypotheses have been proposed to account for the difference between perception and memory estimates. 
One is the reperceptual hypothesis (Kerst \& Howard, 1978; Moyer, Bradley, et al., 1978). According to this hypothesis, when information is stored in memory, any distortions that are a result of the perceptual process are incorporated into the memory trace. During memory retrieval, the memory trace is scanned in a manner analogous to the perceptual process. Therefore, any distortion accrued during the original perceptual encoding is now increased so that the exponent of the memory function should be the square of the perception function. Kerst and Howard tested this hypothesis using a distance-estimation task. The subjects' task was to estimate the distance from the centers of two noncontiguous states (e.g., Utah and Ohio). On the basis of their data, they argued for the reperceptual hypothesis. In their study, the perception exponent was slightly larger than unity (1.04). If the memory estimates were based on a reperceptual process, then one would expect the memory exponent to be $(1.04)^{2}$, or 1.08 . This prediction was supported; the memory exponent was 1.1.

However, there are some aspects of Kerst and Howard's (1978) method that may limit the reperceptual hypothesis. First, they were relying on a spatial configuration with which subjects had had prior experience. This prior experience could have led to highly accurate estimates of distance in both the perception and memory conditions. Second, the exponents for the perception and memory conditions were quite close. There was no statistical test comparing these two conditions, and therefore it is uncertain as to whether they are reliably different from each other. Third, in the case of states of the union, the presence of intervening items (other states) could have altered subjects' estimates. It is known from other research (e.g., A. Stevens \& Coupe, 1978) that people tend to organize the states of the union in a hierarchical fashion and at least partially derive spatial estimates from that representation. Fourth, finding a memory exponent that is larger than the perception exponent in a distance-estimation task is unusual-memory exponents are typically smaller than perception exponents. Furthermore, Wiest and Bell (1985) found that the perception exponent averaged around 1.08 across several studies. Based on the reperceptual hypothesis, the memory exponent should be approximately 1.17. Instead, according to Wiest and Bell, the average over many studies was 0.91 . This is in the direction opposite to that predicted by the reperceptual hypothesis.

A second potential explanation for the difference in the psychophysical functions for perception and memory is that the information in the memory trace itself is gradually transformed or compressed over time (Kemp, 1988). According to this transformation hypothesis, memory for spatial information is slowly changed so that it conforms to more schematically consistent values, such as Gestalt principles or hierarchical structures. Over time, the change in the exponent for the memory function reflects changes in the remembered values as they shift to become more consistent with a more schematic representation. Thus, the change in the remembered values is not a result of memory loss, but is a result of an active transformation of the information in memory. Such transformations appear to be a common phenomenon of information stored in longterm memory (e.g., Bartlett, 1932).

Although the first two hypotheses claim that the difference between perception and memory estimates of distance is due to distortions in perceptual encoding or in memory storage, a simpler explanation based on a guessing process during retrieval is possible. The decrease in the memory exponent could be a result of people's being less certain under memory conditions than under perception conditions. This is called the uncertainty hypothesis. Such uncertainty could arise from a number of sources, and there are three versions of the hypothesis that can be considered. The first was outlined by Kerst and Howard (1978). Their version argued that memory estimates may become distorted as a result of a constriction of the response dimension, with subjects being unwilling to use the upper and lower bounds of the response scale. As such, the distortion in memory estimates would be due to a response bias.

A second version of the uncertainty hypothesis was outlined by Algom et al. (1985; Algom, 1992). According to this view, distortions in memory estimates are due to a change in the stimulus dimension. Under memory conditions, there is less certainty concerning the actual range of stimulus values. As a result, consistent with Teghtsoonian's $(1971,1973)$ description of the psychophysical relation of dynamic ranges, as the stimulus range becomes expanded, the exponents of the derived functions decrease.

We propose that a third version of an uncertainty hypothesis can be considered. According to this third view, uncertainty arises when information has been lost from the memory trace, cannot be recovered for some reason, or was never encoded in the first place. This third version is like the first in that it predicts that there should be a tendency for subjects to constrict the response dimension. However, this view claims that, rather than being unwilling to use the extreme ends of the response dimension, subjects are forced into this position when they cannot recover the needed pieces of information from the memory trace. When some information is missing, distance estimation occurs by using what knowledge is available, such as the range of possible differences and the general location of the items in the display, to derive an estimate. While there are meaningful differences between the different versions of the uncertainty hypothesis, they are not considered here but will be in the general discussion. Until then, the focus of the paper is on comparing our third version of the uncertainty hypothesis with the reperceptual and transformation hypotheses.

In cases of greater certainty, we presume that a person has a rather large amount of information available concerning the magnitude to be estimated. As a result, their estimates of the actual magnitude should be more accurate and more closely resemble estimates of spatial magnitude made under conditions where the information is perceptually available. In cases of uncertainty, educated guesses are made about the actual magnitude of the stimulus dimension. The uncertainty hypothesis predicts that responses should show a tendency to avoid extremely large 
and extremely small responses, and instead favor more moderate responses; such a strategy would result in a smaller exponent. This is similar to the proposal made by S. S. Stevens and Greenbaum (1966) that subject estimates tend to regress toward the mean.

In addition to the change in the exponent values, there are other aspects of the data that are predicted by the uncertainty hypothesis. Under memory conditions, the variability of points around the regression line fitting estimated distances against true distances should increase, leading to lower $r^{2}$ values, relative to comparable perception conditions. Furthermore, as the slope of the function becomes less steep, the $y$-intercept should become greater, provided the approximate range of values is known. This is because there is both a decrease in estimates for larger magnitudes and an increase in estimates for smaller magnitudes, thus decreasing the slope of the line and raising the $y$-intercept. In an extreme situation where there is complete guessing, the function should be flat, the $y$-intercept should be equivalent to the mean of the magnitudes, and $r^{2}$ should equal 0 . There is some evidence in the literature that is consistent with these predictions, although not directly supporting it. For instance, both Björkman et al. (1960) and McNamara and LeSueur (1989) found that, on a memory test, subjects showed a tendency to overestimate short distances and underestimate long distances.

One of the most direct means of assessing certainty is to ask subjects to provide confidence ratings of their magnitude estimates. The uncertainty hypothesis predicts that the magnitude-estimation functions should vary with subjects' confidence. Specifically, high-confidence estimates should result in functions that more closely resemble the perceptual functions, whereas low-confidence estimates should result in more deviant functions. This prediction is inconsistent with both the reperceptual and the transformation hypotheses.

The reperceptual hypothesis, as it is stated, makes no predictions concerning the influence of subjects' certainty on their distance estimates. According to the reperceptual hypothesis, distortions in magnitude estimation from memory are a result of the distortion of the perceptual process that has twice influenced the estimation process, once at encoding and again when the same processes were applied to the resultant memory trace. While there may be some role of confidence overall in terms of the variability of the estimates, a minor modification of the reperceptual hypothesis would predict that the estimates that should come closest to the square of the perception estimates would be the high-confidence memory estimates. Such a modification would be a mixed model including aspects of both the uncertainty and reperceptual hypotheses.

According to the transformation hypothesis, distortions are due to changes in the memory trace itself toward a more schematic representation. These changes are described as being general shifts in a specific direction. In regard to distance estimation, the general shift causes the psychophysical function to be more compressive with increased change, as supported by an increase in distortion across time. There is also no provision in the transformation hypothesis for differences in confidence levels. Changes in confidence levels are presumably due to the availability of information at the time of the estimate, not to the degree of schematization that has occurred. According to the transformation hypothesis, distortions are more a function of retention time than of subjects' certainty.

The uncertainty hypothesis also predicts change across time. It is well known in memory research that, over time, detailed information typically becomes less available. While the detailed information is less available, the uncertainty hypothesis does not predict that any of the remaining stored information itself has undergone change. The loss of information over time may result in an increase in the degree of uncertainty on the part of the subject. The uncertainty hypothesis goes further to state how variations in subject confidence levels reflect the accuracy of their estimates. In particular, high-confidence estimates reflect more accurate estimates than do low-confidence estimates.

While it is of interest to understand how distance estimates are influenced by the need to rely on a single memory trace, it is often the case that people are required to estimate the distance between two locations when the spatial information concerning those locations is presented at different times. Such conditions are referred to here as integration conditions. Integration conditions are considered to be cases in which information about the locations of objects within a single perceptual field is presented at different times, which means that it must be integrated in memory. In addition, to make a distance estimate on the basis of these locations, an inference must be made on the basis of the new integrated spatial representation. This estimate is considered an inference because the information was never directly present in the stimulus display. Under integrative conditions, subjects must not only remember the positions of objects in the display, but must also build up a representation of the different positions presented at different points in time, and, at the time of test, make an inference concerning the distance between objects. The errors found under memory conditions should be further increased in these cases because there is a greater possibility of uncertainty. According to Wiest and Bell (1985), integrative conditions typically yield an exponent of 0.75 , which is substantially lower than the average estimates for perception and memory conditions.

The intent of this paper is to suggest that memory distortion in distance estimation, specifically the compressive slope of the power function, follows from failures to accurately retrieve (i.e., the forgetting of the exact representation of the distance, rather than from reperception or memory transformation. In some cases, people must guess, to some degree, what the distance was by relying on what little information they do have available. These differences in the availability of information in short-term memory should be observed in the subjects' confidence in their responses. Specifically, high-confidence responses should more closely resemble perception estimates, whereas low- 
confidence responses should deviate toward some mean response.

\section{PILOT EXPERIMENT}

The pilot experiment helped to establish presentation and retention intervals that produced memory distance estimates that deviated from the perception estimates.

\section{Method}

Stimuli and Apparatus. The study was conducted on a Macintosh IIx computer with a monochrome $(640 \times 480$ pixel) monitor. The edges of the monitor and CPU case were covered with a black cloth to reduce the availability of nondisplay cues that could be used in estimating distances. Also, testing was done under dim lighting conditions and a filter was placed over the screen to reduce effects of phosphor persistence in memory (pilot experiment and Experiment 1 ) and integration (Experiment 2) conditions.

The stimulus display was a square ( 420 pixels on each side) white display field surrounded by a black border. As shown in Figure 1, the display field was divided into an imaginary $7 \times 7$ unit grid, where one unit equaled 60 pixels. The center column and row were used to further divide the field into four quadrants. Thus, each quadrant was a $3 \times 3$ unit square located at the corners of the grid. This division of the display, which was not visible to subjects, was used to place stimulus objects on the screen.

The stimulus objects were the letters $\mathrm{H}, \mathrm{T}, \mathrm{U}$, and $\mathrm{Y}$. Each letter was 56 pixels at the highest and widest points and was displayed in black. Each letter was placed in one of the four quadrants, with one letter appearing per quadrant. Each letter consistently appeared in the same quadrant, with $\mathrm{T}$ in the upper left, $\mathrm{U}$ in the upper right, $\mathrm{H}$ in the lower left, and $Y$ in the lower right quadrant. This placement was used to reduce problems of memory for object identity that could artificially reduce the accuracy of subjects' distance estimates. Within each quadrant, each letter was centered within one of the nine 1-unit squares.

There were 12 Euclidean distances (i.e., $\sqrt{x^{2}+y^{2}}$ ) used in the pilot experiment. These were selected from the set of 24 possible distances obtained from the letter-placement methodology used.

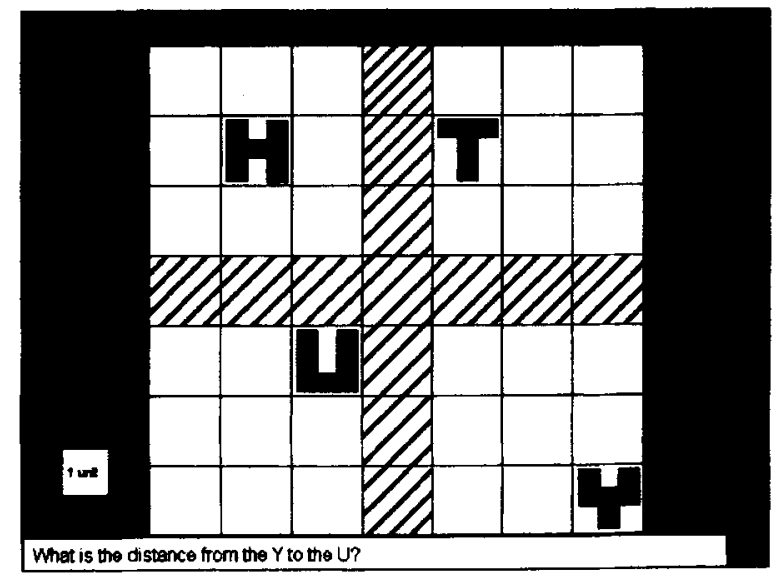

Figure 1. Sample display screen of the type used in all of the experiments. The screen display included a large white display field with four letters, a unit box that was the unit of measurement to be used, and a question box beneath the display field. The lines in the display field were not presented to the subjects, but are presented in the figure to show how the display field was partitioned for letter placement. The shaded region was a no-man's-land that never contained any letters.
These distances were $3.00,4.24,4.74,6.00,6.36,7.50,8.09,8.75$, $9.12,9.60,10.60$, and 11.72 units of 60 pixels. On each trial, two letters were designated as probe letters. These letters were separated by the critical distance for that trial. The remaining two letters were randomly placed within their appropriate quadrants, thereby serving as distractors. The assignment of the probe letters for each trial was determined randomly. For example, in Figure 1, the probe letters are $T$ and $\mathrm{H}$ and the distractors are $\mathrm{U}$ and $\mathrm{Y}$.

Procedure. Subjects were told that they would be participating in an experiment on distance estimation. They were told that their estimates should be the distance from the center of one letter to the center of another. Accuracy was emphasized.

The trials were divided into two conditions, perception and memory, and these were intermixed. In the perception condition, the display remained present through the entire trial. On the memory trials, the display was presented for one of five presentation intervals: 0.5 , $1,5,20$, and $60 \mathrm{sec}$. After the presentation interval, the letters were removed; this was followed by one of five retention intervals: $0,0.5$, 3,15 , and $60 \mathrm{sec}$. During the retention interval, only the empty display field was present.

Immediately for the perception condition and at the end of the retention period for the memory condition, a $640 \times 28$ pixel white question box appeared beneath the display field. The distance-estimation questions were placed in this box. The subjects were asked "What is the distance from the $\mathrm{M}$ to the $\mathrm{N}$ ?" when $\mathrm{M}$ and $\mathrm{N}$ were the appropriate probe letters from the display. The subjects were asked to give their estimates in units, with 1 unit defined as a $40 \times 40$ pixel square. For reference, a sample unit square with the phrase "1 unit" in the center was presented to the left of the display field along with the question. This unit was $2 / 3$ the size of the unit originally used to create displays. The subjects were told to use decimals if they wished.

Each distance was presented once in the perception condition and once per combination of presentation and retention intervals in the memory condition in a mixed fashion, for a total of 26 trials per distance, or 312 trials altogether. In addition to the experimental trials, there were 5 practice trials to familiarize the subjects with the procedure. The subjects were tested in two sessions on different days, with half of the experimental trials being presented on one day and the other half on the other. Each session lasted about $1 \mathrm{~h}$.

Subjects. Ten subjects were tested. They were drawn from the subject pool at Michigan State University and were given partial class credit for their participation.

\section{Results and Discussion}

For each subject, in each condition, the actual distances and the distance estimates were log-transformed and submitted to a regression analysis that provided the exponent, $y$-intercept, and $r^{2}$. This method of analysis was used in all of the experiments reported in this paper. The mean values appear in Tables 1, 2, and 3, respectively.

Because the number of trials per presentation-retention interval were few, any analysis would be tentative, so only general trends are considered. As seen in Table 1, the exponents in all of the memory conditions were decidedly different from those in the perception condition, suggesting that when people need to estimate distances from memory, even at very short retention intervals, their estimates are distorted. The $r^{2}$ data, presented in Table 3, show that estimate variability increased with decreased presentation intervals. The most substantial drop was from 5 to $1 \mathrm{sec}$, so a 3-sec presentation interval was adopted for Experiments 1 and 2 . Also, estimation variability tended to decrease with longer retention intervals. The most substantial drop was between 3 and $15 \mathrm{sec}$, so a 10-sec retention interval was adopted for Experiments 1 and 2. 
Table 1

Mean Perception and Memory-Based Exponents by Presentation and Retention Interval (Both in Seconds) for the Pilot Experiment

\begin{tabular}{ccccccc}
\hline & \multicolumn{7}{c}{ Retention } \\
\cline { 2 - 7 } Presentation & 0 & .5 & 3 & 15 & 60 & $M$ \\
\hline .5 & .795 & .848 & .889 & .776 & .724 & .808 \\
1 & .777 & .752 & .856 & .890 & .817 & .818 \\
5 & .883 & .782 & .858 & .831 & .827 & .836 \\
20 & .885 & .910 & .821 & .854 & .649 & .824 \\
60 & .887 & .872 & .844 & .925 & .850 & .876 \\
$M$ & .845 & .833 & .854 & .855 & .773 & \\
\multicolumn{6}{c}{} \\
\hline
\end{tabular}

Table 2

Mean Perception and Memory-Based $y$-Intercepts by Presentation and Retention Interval (Both in Seconds) for the Pilot Experiment

\begin{tabular}{ccccccc}
\hline & \multicolumn{7}{c}{ Retention } \\
\cline { 2 - 7 } Presentation & 0 & .5 & 3 & 15 & 60 & $M$ \\
\hline .5 & .432 & .215 & .157 & .365 & .489 & .332 \\
1 & .372 & .425 & .223 & .157 & .255 & .286 \\
5 & .191 & .432 & .247 & .293 & .239 & .280 \\
20 & .247 & .148 & .344 & .278 & .751 & .354 \\
60 & .199 & .247 & .315 & .104 & .278 & .229 \\
$M$ & .288 & .293 & .257 & .239 & .402 & \\
& \multicolumn{5}{c}{ Perception $y$-intercept $=.147$} \\
\hline
\end{tabular}

Table 3

Mean Perception and Memory-Based $r^{2}$ Values by Presentation and Retention Interval (Both in Seconds) for the Pilot Experiment

\begin{tabular}{ccccccc}
\hline & \multicolumn{7}{c}{ Retention } \\
\cline { 2 - 7 } Presentation & 0 & .5 & 3 & 15 & 60 & $M$ \\
\hline .5 & .717 & .764 & .809 & .651 & .520 & .692 \\
1 & .767 & .741 & .736 & .688 & .723 & .731 \\
5 & .799 & .743 & .785 & .696 & .803 & .765 \\
20 & .798 & .886 & .770 & .757 & .609 & .764 \\
60 & .825 & .791 & .795 & .810 & .787 & .802 \\
$M$ & .781 & .785 & .779 & .720 & .688 & \\
& \multicolumn{7}{c}{ Perception $r^{2}=.941$} \\
\hline
\end{tabular}

\section{EXPERIMENT 1}

The uncertainty hypothesis was tested directly in Experiment 1 . The subjects were presented with displays like those used in the pilot experiment. The primary differences were that a single presentation and retention interval were used in the memory condition, that subjects rated their confidence in their estimates, and that, rather than using a within-subjects comparison, we assigned the perception and memory conditions to two different groups of subjects. This was done to avoid any strategies that might be developed for one condition to influence performance on the other condition. It was expected that distance estimates would be compressed relative to the perception estimates (e.g., Wiest \& Bell, 1985). In addition, it was predicted that the high-confidence memory estimates would be closer to the perception estimates, whereas the low- confidence memory estimates would demonstrate lower exponents and higher $y$-intercepts.

\section{Method}

Stimuli. The apparatus and stimuli were the same as those used in the pilot experiment, with the exception that, given the letter placement methodology used, all 24 possible distances were employed in Experiment 1 . These distances were $3.00,3.36,4.24,4.50,4.74,5.42$, $6.00,6.18,6.36,6.72,7.50,7.65,8.09,8.49,8.75,9.00,9.12,9.48$, $9.60,10.07,10.61,10.82,11.72$, and 12.74 units of 60 pixels. All distances were presented 16 times per subject in a random order.

Procedure. The subjects were told to estimate the distance between the centers of two letters. Accuracy was emphasized. In addition, the subjects rated their confidence in their estimates using a 1-to-5 scale, with 1 indicating not at all confident, 3 indicating moderately confident, and 5 indicating very confident.

For the memory group, the display was presented for $3 \mathrm{sec}$, after which the letters were removed. There was a $10-\mathrm{sec}$ retention period, during which only the empty display field was present. Then the question box appeared underneath the display field. The distanceestimation and confidence-rating questions were placed in this box. For their confidence ratings, the subjects were asked, "How confident were you in your estimate?" For the perception group, the objects remained on the computer screen throughout the trial and the question box appeared immediately.

In addition to the 384 experimental trials, there were 5 practice trials. Subjects in the memory condition were tested in two sessions on different days, with half of the experimental trials being presented on one day and the other half on the other. Subjects in the perception condition were tested during a single day (because they did not have to contend with fixed presentation and retention intervals). Each session lasted about $1 \mathrm{~h}$.

Subjects. Twelve students from Michigan State University and 13 students from the University of Illinois participated in Experiment 1 in exchange for partial class credit.

\section{Results and Discussion}

The mean $r^{2}$, exponent, and $y$-intercept data for Experiment 1 are summarized in Figure 2 and Table 4 . The plotted functions reflect the mean exponent and $y$-intercepts in each condition. The data from 1 subject in the memory group were dropped since the $r^{2}$, exponent, and $y$-intercept scores for that subject were all greater than 2 standard deviations from the mean of the other subjects.

Perception group. Before comparing the data from the memory group with the data from the perceptual group, we will consider the characteristics of the perception group estimates. Overall, perception estimates were fairly accurate. The exponent was relatively close to $1.00(1.1)$, the $y$-intercept was close to $0(-.3)$, and the $r^{2}$ was very high (.93). Each subject's data were also divided on the basis of a median split of their confidence ratings. While there was no difference in the $r^{2} s$ for the two confidence levels $(t<1)$, the high confidence responses produced slightly larger exponents $[t(12)=2.08, p<.07]$ and lower $y$-intercepts $[t(12)=4.21]$.

Memory group. Averaged across confidence ratings, the data are consistent with previous findings that have shown that the psychophysical function for distance estimation is compressed relative to perception estimates (e.g., Wiest \& Bell, 1985). Each subject's data were also divided on the basis of a median split of their confidence ratings. The performance for the high-confidence responses 


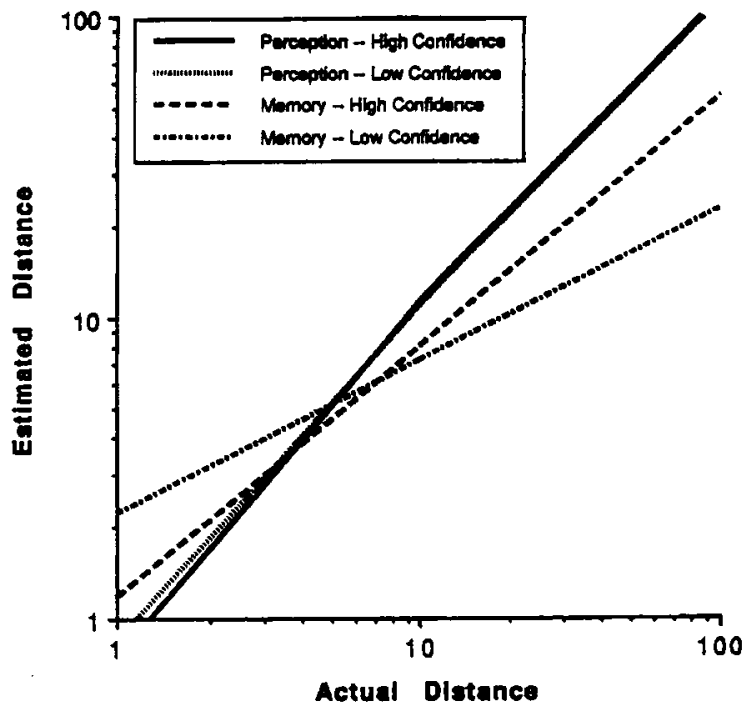

Figure 2. Summary of the data for Experiment 1. The functions for high- and low-confidence perception estimates are very similar. The high-confidence memory function is closer to the perception function than is the low-confidence memory function.

Table 4

Mean $r^{2}$, Exponents, $y$-Intercepts, and Confidence Ratings for Experiment 1

\begin{tabular}{lcccc}
\hline & $r^{2}$ & Exponent & $y$-Intercept & Confidence* \\
\hline Memory & .880 & .702 & .415 & 3.0 \\
High confidence & .864 & .835 & .159 & 4.2 \\
Low confidence & .721 & .509 & .798 & 2.3 \\
Perception & .931 & 1.144 & -.339 & 3.3 \\
High confidence & .914 & 1.167 & -.402 & 3.6 \\
Low confidence & .892 & 1.121 & -.275 & 2.9 \\
\hline
\end{tabular}

*Out of 5 .

was more accurate than that for the low-confidence responses. This difference was confirmed by statistical tests $\left[t(10)=2.20, t(10)=4.36\right.$, and $t(10)=4.21$, for $r^{2}$, exponents, and $y$-intercepts, respectively].

The data from the memory group were compared with the data for the perception group. The memory exponent (.70) was smaller than the perception exponent (1.14) $[t(22)=6.18]$. This is consistent with previous findings. When the data were divided into high-and low-confidence responses, the difference between the perception and memory exponents remained for both the high $[t(22)=4.58]$ and low-confidence trials $[t(22)=7.10]$; the high-confidence responses, however, were clearly closer to the perception condition than were the low-confidence responses. ${ }^{1}$ This is consistent with the predictions of the uncertainty hypothesis, but it is not predicted by the reperceptual or transformation hypotheses.

In addition to the exponent data, the perception $y$-intercept $(-.339)$ was substantially lower than the memory $y$-intercept $(.415)[t(22)=5.51]$. Like the exponent data, this difference remained for both the high- $[t(22)=3.72]$ and the low-confidence trials $[t(22)=7.07]$. However, it is clear that the high-confidence $y$-intercept is much closer to the perception $y$-intercept, whereas the low-confidence $y$ intercept is higher; this pattern is consistent with an overestimation of short distances and an underestimation of long distances, or regression toward the mean, as predicted by the uncertainty hypothesis.

In terms of $r^{2}$, overall there was no difference between the memory and perception groups $[t(22)=1.23, p>.20]$ or for the high-confidence data considered alone $[t(22)=$ $1.24, p>.20]$. However, for the low-confidence data, the memory group $r^{2}$ tended to be smaller than the perception group $[t(22)=1.85, p<.08]$. Finally, there was no overall difference in the confidence ratings between the memory and perception groups $(t<1)$. This may be due to the fact that both groups were basing their confidence ratings on the relative difficulty of trials within the particular condition that they experienced, as would be the case given that the manipulation was between subjects.

In sum, the results of Experiment 1 support the uncertainty hypothesis. In particular, distance estimates showed a tendency to regress toward a mean distance under conditions of low confidence but were more similar to perception estimates under conditions of high confidence. This suggests that high-confidence estimates were based less on a guessing process than were low-confidence estimates. In other words, deviations in distance estimation seem to be due more to differences in memory accessibility or availability than to a change in the mental representation itself or to the manner in which information was extracted from the representation. To explore this issue further, we tested whether the same findings would be obtained under integrative presentation conditions as well as memory conditions.

\section{EXPERIMENT 2}

In Experiment 2, subjects were presented with a display in which the objects were revealed at different points in time. Three methods were used. In one, pairs of objects were flashed in the display field at different times. In the other two, the objects were viewed through an aperture so that the entire display was presented in a piecemeal fashion. In one case, the aperture was moved across the display field whereas, in the other, the aperture remained stationary while the display field was moved behind it.

These three conditions were presented to different groups of subjects. For all groups, there were two types of letter pairs in each display: integrative pairs that required integration across time and memory pairs that did not. One group of subjects was in the flashing condition. ${ }^{2}$ These subjects were allowed to view the entire empty display field at all times, and the target objects were flashed in pairs. Distance estimates made between objects presented in different pairs constituted the integrative condition; estimates between objects presented in the same pair constituted the memory condition. In the flashing condition, subjects were able to use both the stable position of the objects in space as well as the framework of the display field to aid in their distance estimates. Another group of subjects participated in the moving-window condition. These 
subjects viewed the display field through a window or aperture that moved across the screen, revealing the objects behind it as it passed over them. Estimates of the distance between objects revealed at different times constituted the integrative condition; estimates between objects revealed at the same time constituted the memory condition. In the moving-window condition, subjects were able to rely on the stable positions of the objects in space but not to use the framework of the display field to aid in their distance estimates. A third group of subjects participated in the moving-background condition. These subjects viewed the display field through a window that remained stationary in the middle of the screen while the display field was passed behind it. Like the moving-window condition, distance estimates made between objects revealed at different times constituted the integrative condition, while estimates made between objects presented at the same time constituted the memory condition. In the moving-background condition, subjects could rely on neither the framework of the display field nor the stable position of the objects in space for help in making their distance estimates.

These three conditions progressively removed the amount of spatial information available for creating a representation in memory. The presence of reference points, such as the display-field borders or a fixed position in space, has been shown to influence distance estimation (e.g., Holyoak \& Mah, 1982; Sadalla, Burroughs, \& Staplin, 1980). As a result, as the amount of spatial information decreases, subjects should need to rely upon guessing strategies more often and estimates should become increasingly inaccurate.

\section{Method}

Procedure. Subjects were presented with the same displays as in Experiment 1 . The primary difference was in how the displays were revealed. For the flashing condition, the entire empty display field was visible and pairs of objects were placed on the screen for $1 \mathrm{sec}$ each with $3 \mathrm{sec}$ intervening between pairs.

For the moving-window condition, the display field was blackened out except for a window that would reveal a portion of the display field. The objects were revealed as the window passed over them. The window moved either horizontally or vertically across the display field, depending on which edge of the display field it was aligned with. The trials were counterbalanced so that the window began equally often at each edge. On trials where the window began at either the right or left edge, the window was 60 pixels wide and stretched across the entire height of the display field (460 pixels). Similarly, on trials where the window began at either the top or bottom edge, it was 60 pixels high and stretched across the entire width of the display field (460 pixels). On each trial, the window moved slowly across the display field once. It took $6 \mathrm{sec}$ for the window to traverse the length of the display field.

For the moving-background condition, the display field was again blacked out except for a $60 \times 460$ pixel window that was placed in the center of the screen. On each trial, the display field background was moved behind the stationary window. The window was oriented vertically on half the trials and horizontally on the rest. Furthermore, the trials were counterbalanced so that the background was revealed beginning at each edge of the display field equally often. It took $6 \mathrm{sec}$ to move the entire display field behind the window on each trial. At the end of each trial, subjects in all conditions were asked to estimate the distance between the target objects and to give a confidence rating.

We also chose to compare distance estimations that were in the direction of the movement of the window or not. For this reason, all of the experimental distances between objects were either vertical or horizontal. This adds another feature to the data collected. Distance estimates that are made in the direction of the movement are based on objects that do not appear together. In the flashing condition, these are objects that appear in separate pairs. Therefore, integrative processing must be done to derive these estimates. Such conditions are called integrative pairs. In contrast, distance estimates that are not made in the direction of the window movement where both objects appear at once in a window or are flashed together on the screen do not require integrative processing. These estimates are derived only from memory. Such conditions are called memory pairs. Integrative and memory pairs were intermixed randomly throughout the experiment.

Subjects. Thirty-six students from Michigan State University participated in Experiment 2 for partial class credit, with 12 subjects in each presentation condition.

\section{Results and Discussion}

The mean $r^{2}$, exponents, and $y$-intercepts for Experiment 2 are presented in Table 5 . For easy comparison, the perception data from Experiment 1 are repeated.

Overall performance. Averaged across confidence ratings, the data are consistent with previous findings. The psychophysical function for distance estimation is compressive for memory estimates relative to perception estimates and even more so for integration estimates. The exponent was smaller for integrative pair estimates (.563) than for memory pair estimates $(.841)[F(1,32)=29.84$, $\left.M S_{\mathrm{e}}=0.089\right]$. Furthermore, confidence ratings were higher for memory pair estimates (3.4) than for integrative pair estimates (3.3) $\left[F(1,32)=18, M S_{\mathrm{e}}=0.021\right]$.

Table 5

Mean $\boldsymbol{r}^{2}$, Exponents, $\boldsymbol{y}$-Intercepts, and Confidence Ratings for Experiment 2

\begin{tabular}{|c|c|c|c|c|}
\hline & $r^{2}$ & Exponent & $y$-Intercept & Confidence* \\
\hline \multicolumn{5}{|c|}{ Flashing } \\
\hline Memory pair & .967 & .932 & -.025 & 3.3 \\
\hline High confidence & .956 & 1.024 & -.184 & 3.8 \\
\hline Low confidence & .885 & .781 & .289 & 2.8 \\
\hline Integration pair & .911 & .642 & .544 & 3.2 \\
\hline High confidence & .895 & .795 & .216 & 3.7 \\
\hline Low confidence & .638 & .416 & .913 & 2.7 \\
\hline \multicolumn{5}{|c|}{ Moving Window } \\
\hline Memory pair & .963 & .846 & .118 & 3.2 \\
\hline High confidence & .868 & .840 & .136 & 3.7 \\
\hline Low confidence & .869 & .797 & .540 & 2.7 \\
\hline Integration pair & .884 & .598 & .692 & 3.1 \\
\hline High confidence & .828 & .693 & .191 & 3.7 \\
\hline Low confidence & .766 & .506 & .834 & 2.5 \\
\hline \multicolumn{5}{|c|}{ Moving Background } \\
\hline Memory pair & .904 & .822 & .203 & 3.6 \\
\hline High confidence & .875 & .882 & .219 & 4.2 \\
\hline Low confidence & .749 & .717 & .478 & 3.0 \\
\hline Integration pair & .755 & .490 & .620 & 3.4 \\
\hline High confidence & .718 & .561 & .273 & 4.0 \\
\hline Low confidence & .545 & .412 & .752 & 2.9 \\
\hline \multicolumn{5}{|c|}{ Perception $\dagger$} \\
\hline Overall & .931 & 1.144 & -.339 & 3.3 \\
\hline High confidence & .914 & 1.167 & -.402 & 3.6 \\
\hline Low confidence & .892 & 1.121 & -.275 & 2.9 \\
\hline
\end{tabular}

*Out of 5 . †From Experiment 1. 
The $r^{2}$, exponent, and $y$-intercept scores were submitted to separate 3 (group: flashing/moving window/moving background) $\times 2$ (confidence: high vs. low) $\times 2$ (pair type: memory vs. integrative) mixed ANOVAs. The first variable was between subjects, and the rest were within. In general, performance was more accurate for the high-confidence responses than for the low-confidence responses. Consistent with the uncertainty hypothesis, higher confidence responses produced higher $r^{2}$ values $[.857$ vs. . $740 ; F(1,32)=$ $\left.18.12, M S_{\mathrm{e}}=0.025\right]$, exponents that were closer to unity $\left[.800\right.$ vs. $\left..603 ; F(1,32)=34.22, M S_{\mathrm{e}}=0.039\right]$, and $y$ intercepts that were closer to $0[.212$ vs. $.565 ; F(1,32)=$ $\left.34.42, M S_{\mathrm{e}}=0.123\right]$. There were no significant effects involving group.

These data were compared with those of the perception group of Experiment 1. The results are consistent with the notion that both memory and integrative distance estimation are more difficult than perception distance estimation and that integrative distance estimation is more difficult than memory distance estimation. Furthermore, high-confidence estimates were more accurate than low-confidence estimates. Each of the presentation conditions is considered separately. The analyses for each of the confidence ratingpair type conditions are presented in Table 6 .

Flashing group. The data for the flashing group are summarized in Figure 3. The exponents for the high-confidence responses were closer to those for the perception group than were the exponents for low-confidence responses, and for memory pairs relative to integrative pairs. Except for the high-confidence memory pairs, all conditions were significantly different from the perception group. The same pattern of results was replicated for the $y$-intercept data. For the $r^{2}$ data, the only significant difference from the perception group was in the low-confidence integrative condition. This indicates that, except for the most difficult condition (low-confidence integrative), there appeared to be little increase in estimate variability in memory and integrative tasks. There were no significant differences in the reported confidence levels.

Moving-window group. The data for the movingwindow group are summarized in Figure 4. For this group, like the flashing group, the exponents were closer to the perception group for the high-confidence responses than for the low-confidence responses and for memory pairs relative to integrative pairs, although all conditions significantly differed from the perception group. The same pattern of results was replicated for the $y$-intercept data. This suggests that the removal of the display field frame decreased the amount of spatial information available, making the task more difficult and further hampering integrative estimates. Also, like the flashing group, only the most difficult condition (low-confidence integrative) produced $r^{2} \mathrm{~s}$ that deviated significantly from those for the perception group.

Moving-background group. The data for the moving-background group are summarized in Figure 5. For this group, like the flashing and moving-window groups, the exponents were closer to the perception group for the high-confidence responses relative to the low-confidence responses and for memory pairs relative to integrative pairs, although all conditions significantly differed from the perception group. The same pattern of results was replicated for the $y$-intercept data. For the $r^{2}$ data, both high- and low-confidence data in the integration condi-

Table 6

Analyses for the Different Confidence Level-Pair Type

Conditions for Experiment 2 Against the

Perceptual Condition from Experiment 1

\begin{tabular}{|c|c|c|c|c|}
\hline & $r^{2}$ & Exponent & $y$-Intercept & Confidence \\
\hline \multicolumn{5}{|c|}{ Flashing } \\
\hline \multicolumn{5}{|l|}{ Memory pair } \\
\hline High confidence & $t(23)=1.50$ & $t(23)=1.66$ & $t(23)=1.24$ & $t<1$ \\
\hline Low confidence & $t<1$ & $t(23)=3.67^{*}$ & $t(23)=2.76^{*}$ & $t<1$ \\
\hline \multicolumn{5}{|l|}{ Integration pair } \\
\hline High confidence & $t<1$ & $t(23)=4.15^{*}$ & $t(23)=3.74^{*}$ & $t<1$ \\
\hline Low confidence & $t(23)=2.98^{*}$ & $t(23)=7.87^{*}$ & $t(23)=6.79^{*}$ & $t<1$ \\
\hline \multicolumn{5}{|c|}{ Moving window } \\
\hline \multicolumn{5}{|l|}{ Memory pair } \\
\hline High confidence & $t(23)=1.27$ & $t(23)=3.14^{*}$ & $t(23)=2.51^{*}$ & $t<1$ \\
\hline Low confidence & $t(23)=1.09$ & $t(23)=3.72^{*}$ & $t(23)=2.74^{*}$ & $t<1$ \\
\hline \multicolumn{5}{|l|}{ Integration pair } \\
\hline High confidence & $t(23)=1.34$ & $t(23)=3.14^{*}$ & $t(23)=4.37^{*}$ & $t<1$ \\
\hline Low confidence & $t(23)=1.88 \dagger$ & $t(23)=8.16^{*}$ & $t(23)=5.99^{*}$ & $t(23)=1.25$ \\
\hline \multicolumn{5}{|c|}{ Moving Background } \\
\hline \multicolumn{5}{|l|}{ Memory pair } \\
\hline High confidence & $t<1$ & $t(23)=3.37^{*}$ & $t(23)=3.00^{*}$ & $t(23)=2.88^{*}$ \\
\hline Low confidence & $t(23)=1.82$ & $t(23)=4.18^{*}$ & $t(23)=3.67^{*}$ & $t<1$ \\
\hline \multicolumn{5}{|l|}{ Integration pair } \\
\hline High confidence & $t(23)=3.25^{*}$ & $t(23)=7.87^{*}$ & $t(23)=6.41^{*}$ & $t(23)=1.95 \dagger$ \\
\hline Low confidence & $t(23)=3.41^{*}$ & $t(23)=7.11^{*}$ & $t(23)=6.12^{*}$ & $t<1$ \\
\hline
\end{tabular}

${ }^{*} p<.05 . \quad{ }^{\dagger} p<.08$. 


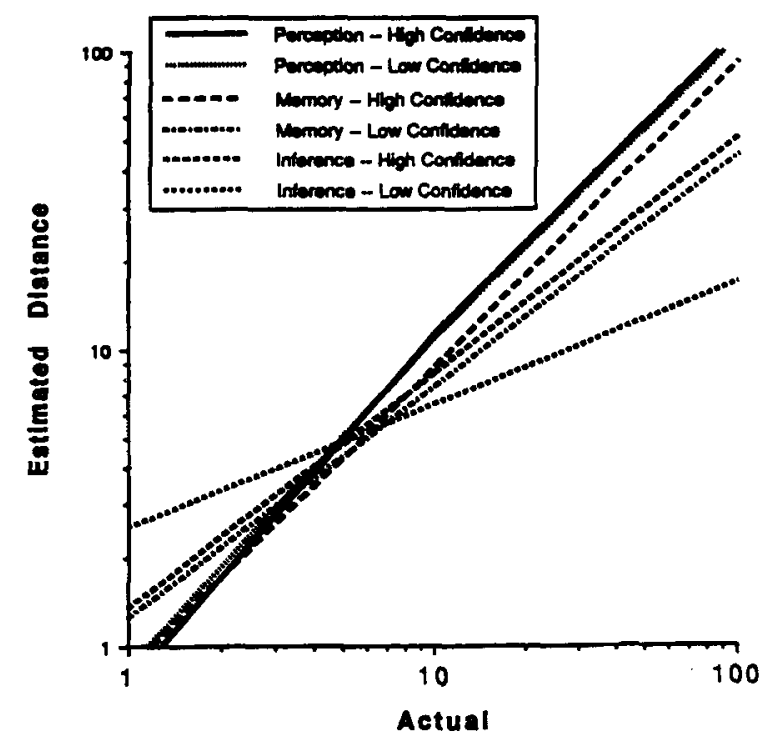

Figure 3. Summary of the data for flashing group in Experiment 2. The perception functions are reproduced from the Experiment 1 data. The experimental data are divided into memory and integrative conditions, as well as high- and low-confidence estimates.

tions significantly differed from those of the perception group. These results suggest that the removal of both the display field and the moving spatial positions of the objects hampered distance estimation for both integrative estimates as well as the encoding process needed for memory estimates. Unlike the flashing and moving-window groups, the confidence ratings in the high-confidence condition were greater than the high-confidence ratings given by the perception group.

The pattern of results for Experiment 2 supports the uncertainty hypothesis. Although distance estimation was poorer in both the memory and the integrative groups relative to the perception group, these distortions were less for high-confidence estimates than for low-confidence estimates across different display conditions. Furthermore, as the reference sources were removed, distance estimation became more difficult. Accuracy was highest when both the display field was present and the objects were stationary in space (flashing), became more distorted when the display field was removed (moving window), and was worst when both the entire display field was not present and the objects were not stationary in space (moving background).

The reperceptual hypothesis is not able to explain the obtained results because the memory pair exponents are variable with respect to the encoding conditions and are not equal to the square of the perception condition. Furthermore, both the reperceptual and the transformation hypotheses are unable to account for the change in the pattern of data from responses of different confidence levels. Finally, neither the reperceptual nor the transformation hypothesis alone provides an explanation as to why distance estimation would be worse under integrative presentation conditions.

\section{GENERAL DISCUSSION}

This paper reports a series of experiments that test the ability of subjects to estimate the distance between objects under a variety of conditions. Performance was best when subjects were able to make distance estimates when the display was perceptually available. Consistent with earlier research (Wiest \& Bell, 1985), accuracy of distance estimation declined when subjects had to estimate distances from memory and was even worse when they had to integrate several pieces of spatial information in order to estimate distances. Furthermore, under integrative conditions, performance was affected by the availability of such spatial cues as the display-field framework or by whether the objects retained a constant spatial position. Finally, distance estimation was better (closer to estimates made under the perception condition) when subject confidence was high.

Two other theories of distance estimation have difficulty in accounting for these results. According to the reperceptual hypothesis (Kerst \& Howard, 1978), distance estimates made from memory undergo the same processing as do perception estimates. As a result, any distortions that result from the perceptual process should be duplicated when the estimates are made from memory. In addition to the lack of evidence that memory exponents equaled the square of the perception exponent, the fact that most of the distance estimates made under both memory and integrative conditions differed from perception estimates more for low-confidence than for high-confidence judgments is inconsistent with the reperceptual hypothesis.

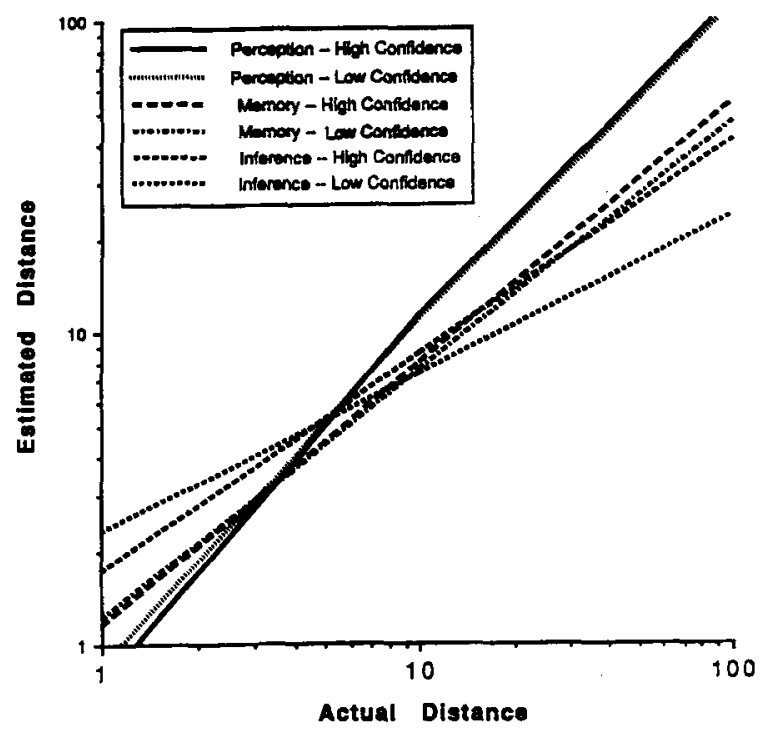

Figure 4. Summary of the data for the moving-window group in Experiment 2. The perception function is reproduced from the Experiment 1 data. The experimental data are divided into memory and integrative conditions and into high-and low-confidence estimates. 


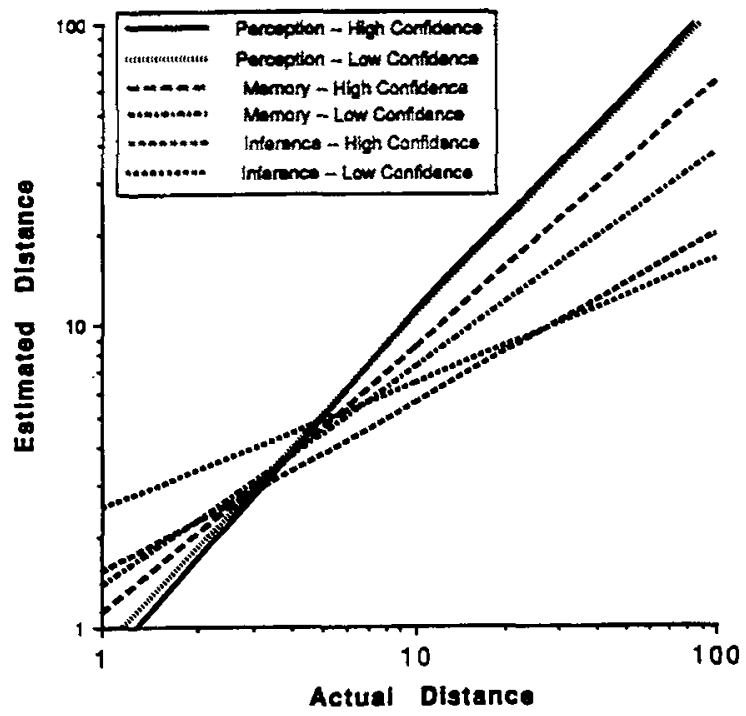

Figure 5. Summary of the data for the moving-background group in Experiment 2. The perception function is reproduced from the Experiment 1 data. The experimental data are divided into memory and integrative conditions and into high- and lowconfidence estimates.

Another, more recent, theory that has been advanced to account for distortions in distance estimation is the transformation hypothesis (Kemp, 1988). This theory accounts for distortions in distance estimation as a result of distortions that occur in a long-term-memory trace over time and is presumed to remain uniform across time. This hypothesis also cannot account for the present data alone because the high- and low-confidence estimates should reflect the same amount of transformation over the same time period.

We favor an uncertainty hypothesis as an explanation for the present data. According to this view, distance estimates from memory are a mixture of highly accurate highconfidence responses and inaccurate low-confidence guesses that are biased by a regression toward the mean. Deviations in estimation are a result of an inability to accurately retrieve all of the information needed to make the estimation rather than to compression in memory. As a result, some guessing about the locations of the objects in space and with respect to one another occurs; such guessing decreases the overall accuracy. We presume that subjects have available some information concerning how much of their estimates are based on an accurate memory representation and how much must be reconstructed in order to provide an estimate, since high-confidence responses were more accurate than low-confidence responses. This may suggest that the New Yorkers' view of the world is not due to compression, but to ignorance-so they lump everything from New Jersey to California in Kansas.

In the introduction we outlined three versions of the uncertainty hypothesis. One version, by Kerst and Howard (1978), argues that uncertainty arises out of subjects' unwillingness to use the entire range of response values. A second version, by Algom et al. (1985), argues that an uncertainty in the stimulus dimension results in compressive functions. Lastly, our own version of the uncertainty hypothesis claims that uncertainty arises out of a loss of information from the memory trace. While it is difficult to distinguish these hypotheses, we prefer our own. A problem with the first version is that, rather than providing an explanation of how the memory representation or estimation process may result in the distortion, it attributes changes in the memory function to a response bias. Our version claims that uncertainty arises out of a loss of information in the memory trace. However, we cannot completely discount the Kerst and Howard version at this time. As for the second version, Algom et al. argue that their view predicts a change in the exponent, and perhaps the $y$-intercept, but not a change in the $r^{2}$ values. However, our data do show some difference between perception and memory $r^{2}$ values.

Although we think that our uncertainty hypothesis provides a better account of the data than do the reperceptual and transformation hypotheses, neither of these explanations can be completely discounted. While both of these models have some credibility, we will focus on the reperceptual hypothesis since it has received more attention than has the transformation hypothesis. The reperceptual hypothesis has been applied to a wide variety of phenomena, from area and distance estimation (Kerst \& Howard, 1978) to labor pains (Algom \& Lobel, 1994).

One of the more notable differences between the current experiments and those that support the reperceptual hypothesis is the fact that studies supporting the reperceptual hypothesis deal with well-learned magnitudes that are tested over very long periods of time. In contrast, the current study supports the uncertainty hypothesis using well-learned magnitudes and involves more of a reliance on short-term memory. As such, it may be that the predictions of the reperceptual hypothesis apply more in welllearned situations in which a person is familiar with the magnitudes, such as estimating the distances between objects in one's own office, whereas the predictions of the uncertainty hypothesis apply more to a situation in which a person is encountered with a novel situation and must estimate the distances between objects, such as the distance between a runner and second base from memory in the absence of instant replay.

From a broader perspective, one framework that could account for the sorts of effects predicted by the uncertainty hypothesis has been proposed by Huttenlocher, Hedges, and Duncan (1991). They argue that systematic errors made in the estimation of physical properties from memory, such as location and distance, are due to a twopart process. One part involves a fine-grained memory of the physical property that stores an unbiased record of the external property. Memory retrieval that involves only this component can be trusted to be unbiased. Unfortunately, due to encoding or retrieval failure, a complete set of information from the fine-grained portion of a memory trace is not always available. In such cases, the information that is available from the fine-grained portion of memory is augmented with information from a categorization process. 
The categorization process involves dividing the stimulus domain along several dimensions. In Huttenlocher et al.'s (1991) study, the domain was location within a circular field. In our study, the domain is distance between objects. When uncertain about a particular stimulus value, because of an impaired fine-grained memory, subjects use the categorization process to assist in the estimation, and report a value that is closer to the prototypical category value. Huttenlocher et al. supported their argument by showing that stimulus items closer to the categorical prototypes resulted in memory estimates that differed less from the actual values than did stimulus items that were near categorical borders. (see also Nelson \& Chaiklin, 1980).

From the perspective outlined here concerning the relative contributions of fine-grained and categorical estimates, certain predictions can be derived and applied to magnitude estimation. First, the degree to which subjects are relying on both a fine-grained memory and a categorization process may be reflected in subjective levels of confidence for provided estimates. Estimates that rely more on fine-grained memories and less on categorization are expected to be higher in confidence, whereas estimates that rely more on categorization and less on fine-grained memories are expected to be lower in confidence. Therefore, when estimates are divided up into high- and lowconfidence reports, higher confidence estimates are closer to perception estimates, if not equivalent to them, whereas lower confidence estimates produce more deviant functions. A second prediction, in line with Huttenlocher et al.'s theory, is that less confident estimates should drift more toward category prototypes. S. S. Stevens and Galanter (1957) have suggested that distance estimation can be influenced by a subject's set of categories of distances. The current research has focused on the first prediction and left this second prediction for subsequent study, because it is both more complicated and depends on establishment of the first prediction, which we were able to confirm here.

\section{REFERENCES}

ALGOM, D. (1992). Memory psychophysics: An examination of its perceptual and cognitive prospects. In D. Algom (Ed.), Psychophysical approaches to cognition (pp. 441-513). New York: Elsevier, NorthHolland.

AlgOM, D., \& LOBEL, S. (1994). Psychophysics in the field: Perception and memory for labor pain. Perception \& Psychophysics, 55, 133-141.

Algom, D., WOLF, Y., \& BergMaN, B. (1985). Integration of stimulus dimensions in perception and memory: Composition rules and psychophysical relations. Journal of Experimental Psychology: General, 114, 451-471.

BARTLETT, F. C. (1932). Remembering. London: Cambridge University Press.

BJörkmAN, M., LundBERG, I., \& TÄRNBLOM, S. (1960). On the relationship between memory and percept: A psychophysical approach Scandinavian Journal of Psychology, 1, 136-144.

CHEW, E. I., \& RICHARDSON, T. E. (1980). The relationship between per- ceptual and memorial psychophysics. Bulletin of the Psychonomic Society, 16, 25-26.

EKMAN, G., \& JUNGE, K. (1961). Psychophysical relations in visual perception of length, area, and volume. Scandinavian Journal of Psychol$o g y, 2,1-10$

HOLYOAK, K. J., \& MAH, W. A. (1982). Cognitive reference points in judgments of symbolic magnitude. Cognitive Psychology, 14, 328-352.

Huttenlocher, J., HedGes, L. V., \& DunCan, S. (1991). Categories and particulars: Prototype effects in estimating spatial location. $P s y-$ chological Review, 98, 352-376.

KEMP, S. (1988). Memorial psychophysics for visual area: The effect of retention interval. Memory \& Cognition, 16, 431-436.

KeRST, S. M., \& HowaRD, J. H. (1978). Memory psychophysics for visual area and length. Memory \& Cognition, 6, 327-335.

McNamara, T. P., Ratcliff, R., \& McKoon, G. (1984). The mental representation of knowledge acquired from maps. Journal of Experimental Psychology: Learning, Memory, \& Cognition, 10, 723-732.

MCNAMARA, T. P., \& LeSueur, L. L. (1989). Mental representations of spatial and nonspatial relations. Quarterly Journal of Experimental Psychology, 41A, 215-233.

Moyer, R. S., Bradley, D. R., Sorensen, M. H., Whiting, J. C., \& MANSFIELD, D.P. (1978). Psychophysical functions for perceived and remembered size. Science, 200, 330-332.

Moyer, R. S., Sklarew, P., \& Whiting, J. (1978). Memory psychophysics. In H. Geissler \& P. Petzold (Eds.), Psychophysical judgment and the process of perception (pp. 35-46). New York: Elsevier, NorthHolland.

NELSON, T. O., \& ChAIKLIN, S. (1980). Immediate memory for spatial location. Journal of Experimental Psychology: Human Learning \& Memory, 5, 529-545.

Sadalla, E. K., Burroughs, W. J., \& Staplin, L. J. (1980). Reference points in spatial cognition. Journal of Experimental Psychology: Human Learning \& Memory, 6, 516-528.

SHEPARD, R. N., \& CHIPMAN, S. (1970). Second order isomorphism of internal representations: Shapes of states. Cognitive Psychology, 1, 1-17.

STEVENS, A., \& COUPE, P. (1978). Distortions in judged spatial relations. Cognitive Psychology, 10, 422-437.

STEVENS, S. S., \& Galanter, E. H. (1957). Ratio scales and category scales for a dozen perceptual continua. Journal of Experimental Psychology, 54, 377-411.

Stevens, S. S., \& Greenbaum, H. B. (1966). Regression effect in psychophysical judgment. Perception \& Psychophysics, 1, 439-446.

TEGHTSOONIAN, R. (1971). On the exponents in Stevens' law and the constant in Ekman's law. Psychological Review, 78, 71-80.

TEGHTSOONIAN, R. (1973). Range effects in psychophysical scaling and a revision of Stevens' law. American Journal of Psychology, 86, 3-27.

THORNDYKe, P. W. (1981). Distance estimation from cognitive maps. Cognitive Psychology, 13, 526-550.

WIEST, W. M., \& BELL, B. (1985). Stevens' exponent for psychophysical scaling of perceived, remembered, and inferred distance. Psychological Bulletin, 98, 457-470.

\section{NOTES}

1. The data from the high- and low-confidence memory conditions were also compared with the overall perception group data, and the results of the statistical tests were unchanged. Therefore, only the tests comparing the high-confidence memory with perception data and the low-confidence memory with perception data are presented.

2. We would like to thank Mike Kelly for suggesting the flashing condition.

(Manuscript received November 15, 1993; revision accepted for publication September 9, 1994 .) 